\title{
Fraud Detection of Mobile App Ranking
}

\author{
Rejin Idiculla Johnson ${ }^{1}$, John Prakash ${ }^{2}$ \\ ${ }^{1}$ PG Scholar, Department of Computer Science \& Engineering, Mangalore Institute of technology and engineering, Mangalore, Karnataka \\ (India) \\ ${ }^{2}$ Associate Professor, Department of Computer Science \& Engineering, Mangalore Institute of technology and engineering, Mangalore, \\ Karnataka (India)
}

\begin{abstract}
Fraud ranking among mobile app's, refers to the fraud activities that happens in the app market. These activities are aimed to bump up the apps in the popularity list. Some developers use shady means, like inflating sales of the app, or post fake apj ting as ranking fraud. More popular apps will get more downloads and will result in developer getting more profit. So far there has-a limited research in this field. This work is about developing a detection system for ranking fraud among mobile apps. The yo $\$$ based on finding the leading sessions of mobile apps, which tells a time range in which the app is mostly used. The next step iry 345 making use of three types of evidences. These are ranking, rating and review evidences. These will be they aggregated andsy pared with data collected from app datasets.
\end{abstract}

Keywords: Ranking Fraud, App Rating and Review, Evidence Aggregation, Leadis
1. Introduction

This is the age of mobile technology. People depend heavily on mobile devices. There exists 1.7 million apps as of noy in Google's play store and Apples app store. Each apps have a specific use and different interface. To promo development of these mobile applications the application stores also known as app markets has leaderboard chart. These apps are ranked in based on their popularity.

An application which will have a higher
list will result in more number of diynl turn will provide a source of $r$ developer uses various ma But since recent times, se

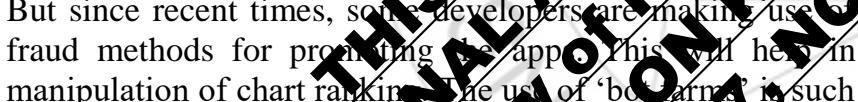

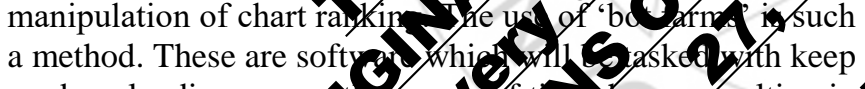

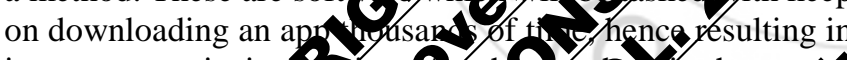
improvement in it

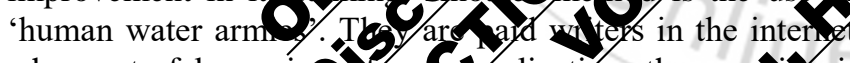
who posts fake revie or. Syplication, thus masy.jo it appear a trusted protuy.

So far there has be finited development

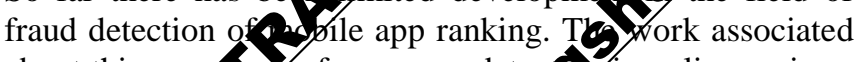
about this area from spam detect in online reviews and web detection. Due to need of a proper detection proponism, the provout a detection system android mobile application. There are several factors Which should be co fored for this. One main factor is the time of popularity of an application. This is the time range in an application is popular and is widely used. This is also the time the application is most likely to be exposed to fraud activities. This time range is called a Leading Session. By identifying this leading session, the ranking pattern of the application can be determined. Further the app's rating and review details given by the users are also taken into account. These details can be compared against previous historical details of the application that has been collected. The \section{Related Work}

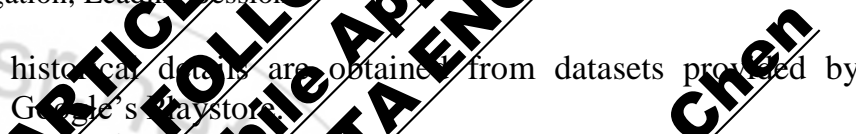
$4 \%$

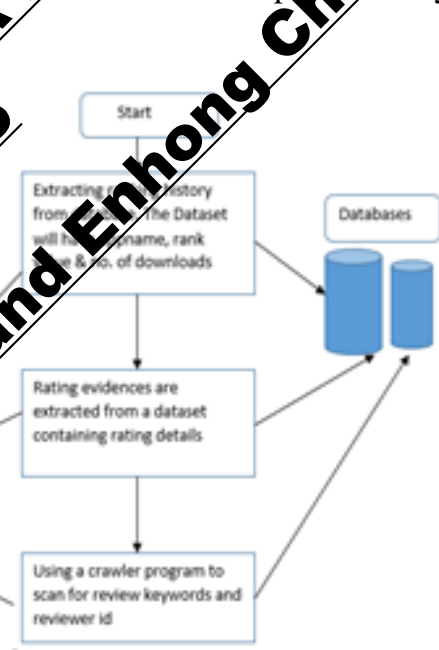

OIgure 1: The Framework for the detection system 1

The first is about web ranking spam detection. It refers to any deliberate actions which bring to selected webpages an unjustifiable favourable relevance or importance. For example, Ntoulaset have studied various aspects of contentbased spam on the web and presented a number of Heuristic methods for detecting content based spam. Zhou have studied the problem of unsupervised webranking spam detection. Specifically, they proposed an efficient online link spam and term spam detection methods using spamicity. Recently, Spirin and Han have reported a survey on web spam detection, which comprehensively introduces the principles and algorithms in the literature. Actually, the work of web ranking spam detection is mainly based on the analysis of ranking principles of search engines, like PageRank and query term frequency. This is different from ranking fraud detection for mobile Apps.

The second category is concentrated on detecting online review spam. For example, Lim have identified several indicative behaviours of review spammers and model these behaviours to detect the spammers. Wu have studied the

Volume 5 Issue 6, June 2016 www.ijsr.net 


\section{International Journal of Science and Research (IJSR) \\ ISSN (Online): 2319-7064}

Index Copernicus Value (2013): 6.14 | Impact Factor (2015): 6.391

problem of detecting hybrid shilling attacks on rating data. The proposed approach is based on the semi-supervised learning and can be used for reliable product recommendation. Xie have studied the problem ofsingleton review spam detection. Specifically, they solved this problem by detecting the co-anomaly patterns inmultiple review based time series. Although some of above approaches can be used for anomaly detection from

Historical rating and review records, they are not able to extract fraud evidences for a given time period.

The third category includes the studies on mobile App recommendation. For example, Yan and Chendeveloped a mobile App recommender system named Appjoy, which is based on user's App usage records to build a preference matrix instead of using explicit user ratings. Also, to solve the sparsity problem of App usage records, Shi and Ali studied several recommendation models and proposed a content based collaborative filter model called EigenApp. Some researchers studied the problem of exploiting enriched contextual information for personalized context aware recommendation which integrates both context dependency and independency assumptions.

\section{Problem Statement and Proposed Solution}

\subsection{Problem Statement}

An app with the higher ranking in the charts downloads. Some app developers will use to deliberately boost their Apps. Some will be usage of bot farms or human the huge number of mobile Apps label ranking fraud for each
nature of chart rankings, confirm the evidences link

\subsection{Proposed Solution}
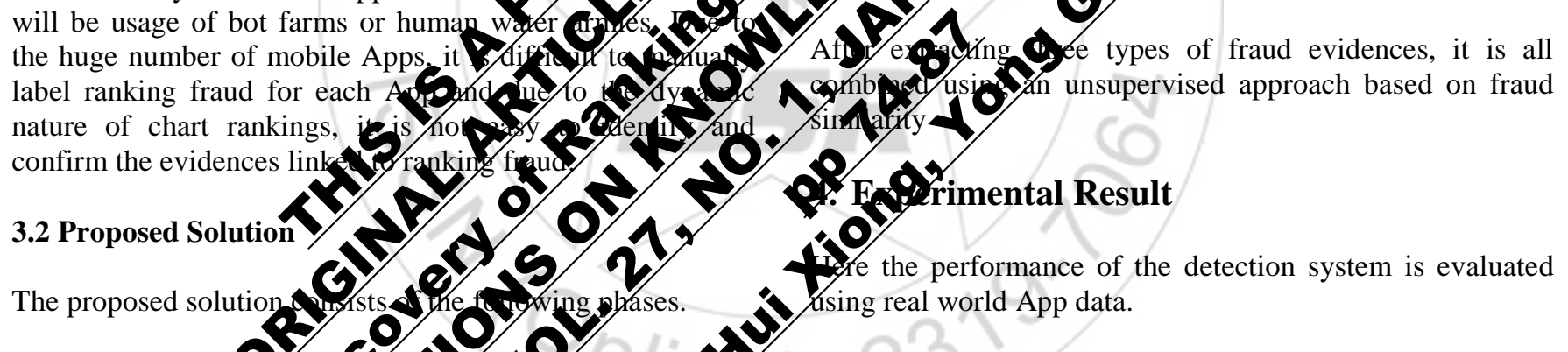

\subsection{Extracting Rating Based Evidence}

The rating evidence is obtained from a dataset containing apps historical details. The user ratings during a time period may have anomaly patterns compared with its historical rating. If an App has ranking fraud in a leading session, the ratings during the time period may have anomaly patterns compared with its historical ratings, which can be used for constructing rating based evidences

\subsection{Extracting Review Based Evidence}

Most of the App stores also permit users to write some textual comments as App reviews. Such rew can indicates the individual perceptions and usage ences of existing users for particular mobile Review manipulation is one of the most valuab erspectives of App ranking fraud. Sroficaty, be downloading or purchasing new nus ally first read its

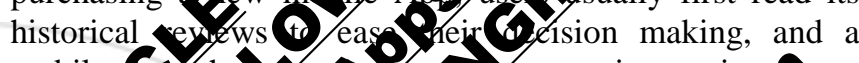
mobile of that ont moncouraging review

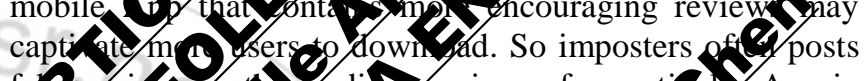

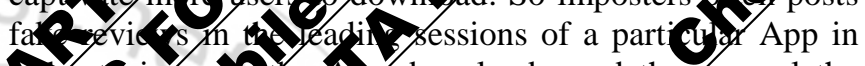

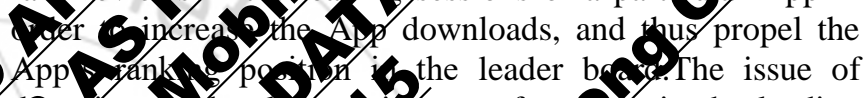
conting the leading detecinon bavis in leading session. 3.3 Extracting RaAing 4.1 The Experimental Data By analysing the Ayps, rorical ranking royts, we observe that Apps' ran behaviours in a ledying event always satisfy a spe ranking pattern, consists of three different ming phases, nam phase, maintaining phand recession phas ands ranking first increases to peak position in leoboard, then keeping such peak yition for a period ranking decreases till end of envent.
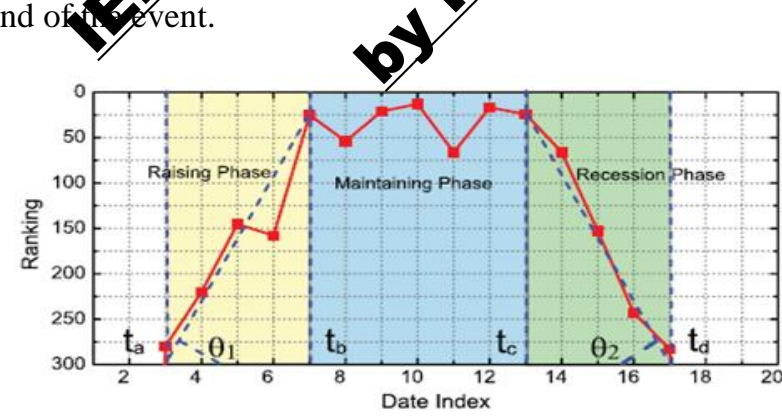

Figure 2: Ranking Pattern of an App
The experimental data sets were collected from the "Top Free 300" and "Top Paid 300" leader boards of Apple's App Store from February 2, 2010 to September 17, 2012. The data sets contain the daily chart rankings 1 of top 300 free Apps and top 300 paid Apps, respectively. Furthermore, each data set also contains the user ratings and review information. Figs.3a and $3 \mathrm{~b}$ indicate the distributions of the number of Apps with respect to different rankings in these data sets. In these figures, we can notice that the number of Apps with low rankings is more than that of Apps with high rankings. Additionally, the competition between free Apps is more than that between paid Apps, especially in high rankings (e.g., top 25). Figs. $4 \mathrm{a}$ and $4 \mathrm{~b}$ show the distribution of the number of Apps with respect to different number of ratings in these data sets. In these figures, we can notice that the distribution of App ratings is not even, which shows that only a small percentage of Apps are very popular. 


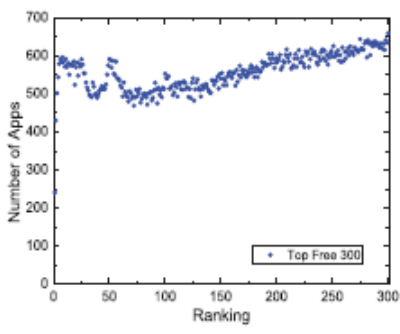

(a) Top Free 300 data set

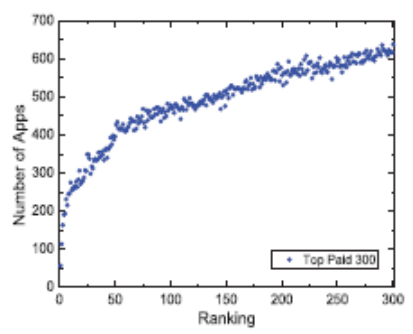

(b) Top Paid 300 data set

Figure 3(a) and Figure 3(b)

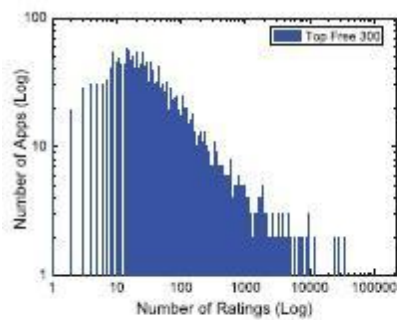

(a) Top Free 300 data set

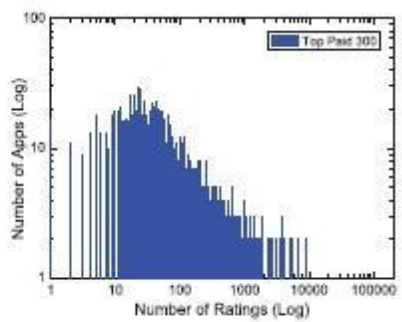

(b) Top Paid 300 data set

Figure 4(a) and Figure 4(b)

[3] S. Xie, G. Wang, S. Lin, and P. S. Yu, "Review spam detection via temporal pattern discovery," in Proc. 18th ACM SIGKDD Int. Conf. Knowl. Discovery Data Mining, 2012

[4] B. Zhou, J. Pei, and Z. Tang, "A spamicity approach to web spam detection," in Proc. SIAM Int. Conf. Data Mining, 2008

[5] A. Ntoulas, M. Najork, M. Manasse, and D. Fetterly, "Detecting spam web pages through content analysis," in Proc. 15th Int. Conf. World Wide Web, 2006

[6] B. Yan and G. Chen, "AppJoy: Personalized mobile application discovery," in Proc. 9th Int. Conf. Mobile Syst., Appl., Serv., 2011

[7] H. Zhu, H. Cao, E. Chen, H. Xiong, and Tian, "Exploiting enriched contextual informatis mobile app classification," in Proc. 21st Int. Conf. Inform. Knowl. Manage, 2012

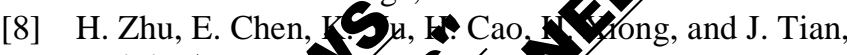

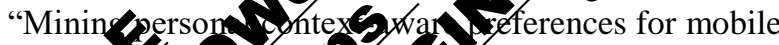
users, Pr YEF th C. Conf. Data Mining, $200 \%$ a $\mathrm{K}$. [9] Keytie D. Kth, and K. Sms\%"An

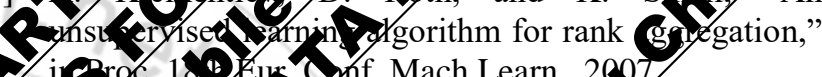

\section{Conclusion}

To develop a ranking fraud detection system for Apps, we first discover that ranking fraud occur sessions and provided a method for mining leadit for each App from its historical ranking recor we identified ranking based evidences evidences and review based evidences fraud. An optimization based integrate all the evidences for leading sessions from mobile the unique perspective of extended with other extended with other
detect ranking fraud.
system with extensive collected from the 10 mor data showed the effectivs of proct

\section{Acknowledga o l}

I take this opportunity Jank Prof. John Prals, Prof. P V Bhat \& Prof. DrNage $\mathrm{N}$, for their valut guidance and for providing an necessary suppor accomplish this research. I youlo/ike to extend my ditude towards our beloved Prgn al G L Eshwar Pras Oor his great support

\section{Refervices}

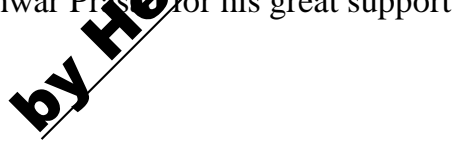

[1] E.-P. Lim, V.-A. Nguyen, N. Jindal, B. Liu, and H. W. Lauw, "Detecting product review spammers using rating behaviors," in Proc. 19th ACM Int. Conf. Inform. Knowl. Manage 2010

[2] Z. Wu, J. Wu, J. Cao, and D. Tao, "HySAD: A semisupervised hybrid shilling attack detector for trustworthy product recommendation," in Proc. 18th ACM SIGKDD Int. Conf. Knowl. Discovery Data Mining, 2012 